DOI: $10.12731 / 2227-930 X-2016-4-37-45$

\title{
IMPLEMENTATION \\ OF INCIDENT DETECTION ALGORITHM BASED ON FUZZY LOGIC IN PTV VISSIM
}

\author{
Nikolaev A.B., Sapego Yu.S.
}

Traffic incident management is a major challenge in the management of movement, requiring constant attention and significant investment, as well as fast and accurate solutions in order to re-establish normal traffic conditions. Automatic control methods are becoming an important factor for the reduction of traffic congestion caused by an arising incident.

In this paper, the algorithm of automatic detection incident based on fuzzy logic is implemented in the software PTV VISSIM. 9 different types of tests were conducted on the two lane road section segment with changing traffic conditions: the location of the road accident, loading of traffic.

The main conclusion of the research is that the proposed algorithm for the incidents detection demonstrates good performance in the time of detection and false alarms

Keywords: Traffic incident management; PTV VISSIM; Automatic methods; detection of road incident.

\section{Introduction}

Application of fuzzy logic in the system of incident detection allows to make a decision under conditions of uncertainty. Process of traffic incidents detection is a process of finding difficulties in traffic, which occur on the roadway. The difficulty in the conveyor flow is a major indication that there was a road accident and its elimination 
requires a reaction. The difficulty in traffic is the main indication that there was a road accident and its elimination requires a reaction.

This conclusion led to the use of input data that will be relevant to the vehicles and the road, with this data must be considered together and should be compared with the corresponding values for further analysis. The main parameters of the traffic flow, which can characterize its current state, are: flow rate; flow volume.

Necessary to analyze the data received from the sensors. After that, the fuzzy rules take these data as evidence, and depending on them and imposed fuzzy rules will be taken action that will improve the situation in traffic or at least not allow it worse.

When an incident occurs, it is formed on the road a congestion. Once the incident is considered to be eliminated, the road capacity is increased and congestion is dissipated. The algorithm determines the state of the road on changing of flow rate and flow volume [11]. In proposed algorithm based on fuzzy logic, the following data are used (Fig. 1).

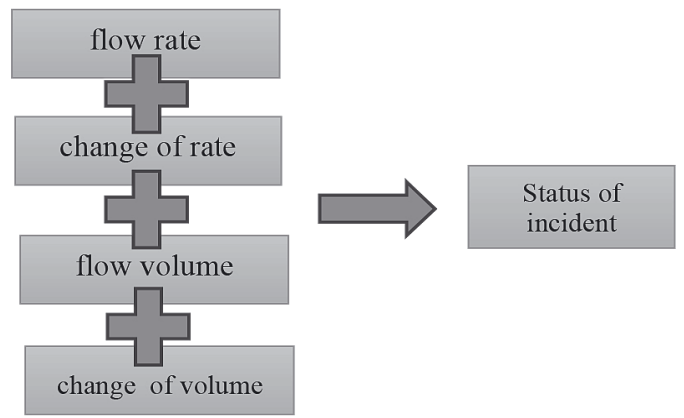

Fig. 1. The input data to determine the status of the incident

Proposed algorithm will produce one of the following results:

1. The traffic is normal.

2. Incident is probably occurred.

3. Incident is found.

The algorithm of the incident detection system is the following (Fig. 2). 


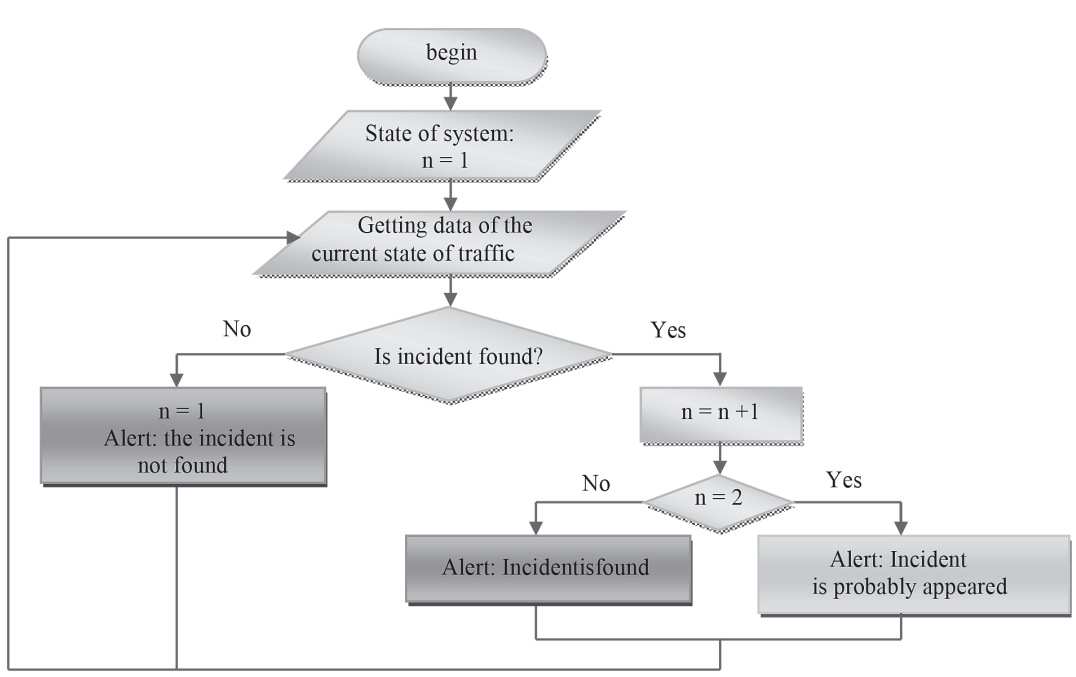

Fig. 2. The algorithm of the incident detection system

During a certain period, current state of traffic will be for occurrence of the incident. If the analysis shows that the traffic is not normal, it is considered likely that the incident occurred (situation 2), or - situation 1. If the situation is over 3 time intervals measured traffic is not normal, it is considered that a road incident occurred, then the output will be situation 3. If the situation has returned to normal flow, so that the incident has been eliminated, and the output will again situation 1. After the system has shown that the incident occurred, the incident classification stage starts.

\section{Determination of efficiency measure of the algorithm}

The effectiveness of traffic incident management system is related especially with the implementation phase of the detection of the incident. The following parameters characterize the effectiveness of incident detection algorithms:

- $\quad D R$ (Detection Rate) - is defined as the percentage of incident cases detected correctly by the algorithm. 


$$
D R=\left(\frac{N_{D I}}{N_{T I}}\right) * 100
$$

where $N_{D I}$ - the number of detected incidents,

$N_{T I}$ - the total number of incidents.

- FAR (False Alarm Rate) - is an index to represent the rate at which the non-incident cases are falsely classified as incident cases.

$$
F A R=\left(\frac{N_{F A}}{N_{T A}}\right) * 100
$$

where $N_{F A}$ the number of false alarms,

$N_{T A}$ - the total number of alarms.

- MTTD (Mean Time to Detection) - it is defined as the time from the moment when the incident occurred until it is discovered.

$$
\text { MTTD }=\sum_{i=1}^{N}\left(t_{a}-t_{\text {inc }}\right) / n
$$

where $N$ - the number of detected incidents,

$t_{a}$ - the time when the incident was discovered,

$t_{i n c}-$ the time when the incident occurred.

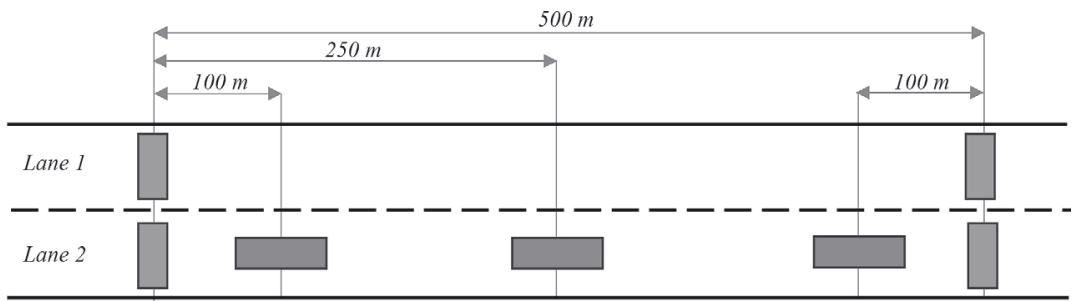

Location of the traffic incident

Detector

Fig. 3. Simulated study area

Detection of the incident can be considered as an essential component of the incident management process. As soon as an incident is detected and verified, and other actions must be launched for the elimination of the existing incident. In order to ensure effectiveness 
of any incident management process it is important that emerged incident was detected as soon as it arose. A timely and accurate incident management becomes more important when one considers the negative impact on road traffic and its safety. The delay in identifying the incident may lead to long traffic jams, which can be a major cause of secondary accidents.

\section{Data Description}

Simulation of the incident and collect data about it will be implemented in software PTV Vissim. Software PTV VISSIM is microscopic simulators stochastic traffic that was used to create a detailed model of I-210 West. In the past, it used mainly as a tool for the design of urban public transport systems, but later was used for the simulation of traffic on the highway.

Vissim has not tools for real model simulations of the traffic incident, so the data will be collected through the creation of on the roadway a parking space that will be considered as a traffic accident.

Installed road options in PTV Vissim (Fig. 3):

- Number of lanes in one direction - 2 lanes;

- Width of the roadway - 3.5 meters (based on the average value of the width of the roadway for the roads usual use (not high speed) of different categories according to GOST R 52398);

- The length of the roadway - $1000 \mathrm{~m}$;

- The distance between the sensors - $500 \mathrm{~m}$;

- The transport stream: motor cars $-95 \%, \mathrm{HGV}-2 \%$, buses $-3 \%$.

Definition of conditions of traffic incident simulation:

- In order to analyze the efficiency of the algorithm in a variety of traffic conditions, road section will be loaded with 3 different traffic load values: 2000 vehicles/h, 4000 vehicles/h, 8000 vehicles/h.

- Incidents (parking spaces) will be established in three different places: 
o At a distance of 100 meters after the first detector.

o Exactly halfway between the detectors (at a distance of 250 meters).

o 100 meters up to the second detector.

The data will be obtained every 30 seconds from the sensors. Incident detection algorithm will be tested at 3 different road conditions (with different traffic loads and with different location of incident).

\section{Experimental Analysis}

The performance of the proposed model has been evaluated by standard parameters (MTTD, DR, FAR), assessing the effects of some factors. 9 various experiments were performed with different traffic conditions in software PTV Vissim:

- Parking space located in three different locations with respect to the installed sensors (after $100 \mathrm{~m}$, in the middle, before $100 \mathrm{~m}$ ).

- Change traffic load values (2000 vehicles/h, 4000 vehicles/h, 8000 vehicles/h).

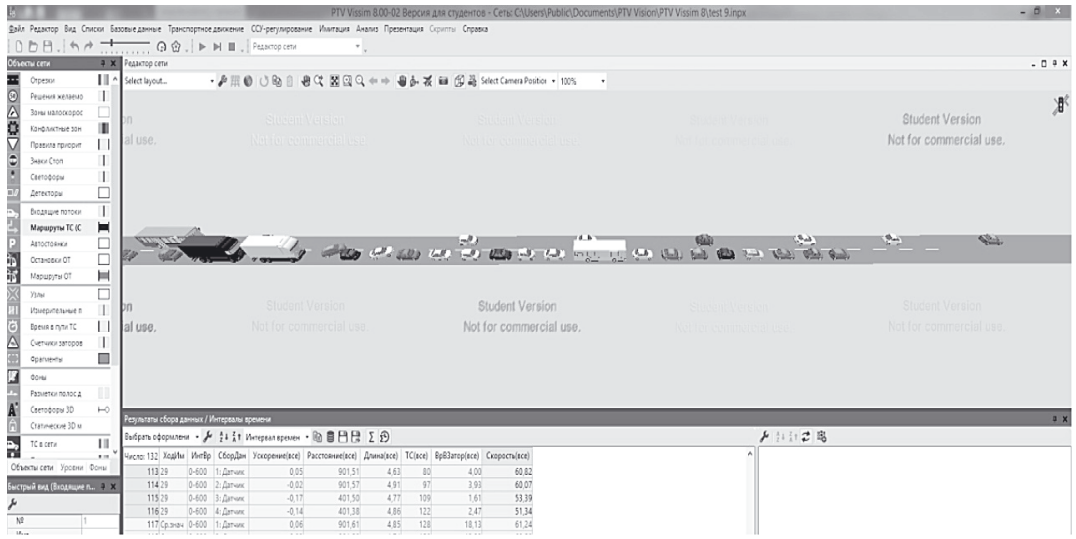

Fig. 4. Screen simulation of traffic when incident occurs

10 situations were simulated in each experiment, in which the incident occurred (Fig. 4). It was analyzed the data before the incident, 
during (as the car took the parking space), after. Below are the average values of the results of all of the experiments (table 1):

Table 1.

Results of all of the experiments

\begin{tabular}{|c|c|c|c|c|c|}
\hline № & $\begin{array}{c}\text { Location } \\
\text { of the incident }\end{array}$ & $\begin{array}{c}\text { Traffic load values, } \\
\text { vehicles } / \mathrm{h}\end{array}$ & MTTD, s & DR, \% & FAR, $\%$ \\
\hline 1 & \multirow{3}{*}{ after $100 \mathrm{~m}$} & 2000 & 63,3 & 100 & 0 \\
\hline 2 & & 4000 & 20,7 & 100 & 0 \\
\hline 3 & & 8000 & 18,3 & 100 & 0,1 \\
\hline 4 & \multirow{3}{*}{ in the middle } & 2000 & 64 & 100 & 0,1 \\
\hline 5 & & 4000 & 36,3 & 100 & 0 \\
\hline 6 & & 8000 & 39,3 & 100 & 0 \\
\hline 7 & \multirow{3}{*}{ before $100 \mathrm{~m}$} & 2000 & 65,3 & 100 & 0 \\
\hline 8 & & 4000 & 31,7 & 100 & 0,1 \\
\hline 9 & & 8000 & 30,3 & 100 & 0 \\
\hline \multicolumn{3}{|c|}{ Total } & 41,02 & 100 & 3,33 \\
\hline
\end{tabular}

The proposed algorithm showed poor results at low loading of traffic (2,000 vehicles) - incident detection time is maximum. Minimum detection time was observed at high loading (8000 cars) - 18.3 seconds, which is caused by the fact that the presence of incident on the road more rapidly affect the overall traffic flow than at low loading.

Several experiments showed false alarms of incidents, two of them were at a loading of 4,000 cars. At higher loading of traffic, the system showed the presence of incident after his elimination. It is caused by the during incident action on the simulated road congestion was formed, which was not disappeared immediately after the removal of incident.

It's observed the following regularities: the more loading of traffic, the less time of detection.

\section{Conclusions}

In this paper, the algorithm is implemented to detect incidents based on fuzzy logic in program PTV VISSIM. 9 various experiments were performed with different traffic conditions in software PTV Vissim: 
- Parking space located in three different locations with respect to the installed sensors (after $100 \mathrm{~m}$, in the middle, before $100 \mathrm{~m}$ ).

- Change traffic load values (2000 vehicles/h, 4000 vehicles/h, 8000 vehicles/h).

According to a result of modeling the effectiveness of the proposed algorithm has been evaluated on three basic indicators (Mean Time to Detection (MTTD), Detection rate (DR), False Alarm Rate (FAR)).

The following conclusions can be drawn based on the results of algorithm performance:

- Mean Time to Detection (MTTD) is 41,02 seconds. This means that the system can detect the presence of incident in two phases. (each phase lasts 30 seconds), which is a very good indicator.

- Detection rate (DR) is high (100\%) even at low loading of traffic.

- False Alarm Rate (FAR) has an average value. The system showed only 3 false signal at 90 test.

\section{Reference}

1. Abdulrahman Alkandari. Accident Detection and Action System Using Fuzzy Logic Theory// Proceedings of 2013 International Conference on Fuzzy Theory and Its Application. Dec. 6-8, 2013. Taipei, Taiwan, pp. 385-390.

2. Cherrett $\mathrm{T}$. Remote automatic detection of the incident using inductive loops. Proceedings of the ICE - Transport. 2005.

3. Deniz O., Celikoglu H.B. Overview to some existing incident detection algorithms: a comparative evaluation // Procedia - Social and Behavioral Sciences. 2011, pp. 1-13.

4. Iancu i. A Mamdani Type Fuzzy Logic Controller// Fuzzy Logic Controls, Concepts, Theories and Applications. University of Craiova Romania, pp. 325-350.

5. Hi-ri-o-tappa K., Likitkhajorn C., Poolsawat A.S. Thajchayapong. Traffic incident detection system using series of point detectors// Intelligent Transportation Systems (ITSC), 15th International IEEE Conference on. 2012, pp. 182-187. 
6. Kaan M.A. Ozbay. Evaluation of incident management strategies and technologies using an integrated traffic/incident management simulation World Review of Intermodal Transportation Research. 2005, pp. 51-82. ISSN: 1749-4737

7. Manstetten D., Maichle J. Determination of traffic characteristics using fuzzy logic. $11 \mathrm{pp}$.

8. Mingwei $\mathrm{Hu}$, Hao Tang. Development of the Real-time Evaluation and Decision Support System for Incident Management. IEEE. 2003, pp. 426-431.

9. Rossi R., Gastaldi M. Fuzzy logic-based incident detection system using loop detectors data// Transportation Research Procedia 10 (2015), pp. 266-275.

10. Rubanov V.G. , Filatov A.G. , Rybin I.A. Intelligent automatic control system. Fuzzy control in technical systems. Electronic manual. URL: http://nrsu.bstu.ru/chap27.html

11. Sapego Yulia, Nikolaev A. Developing incident detection algorithm based on the Mamdani fuzzy inference algorithm.

12. Sergio Mitrovich, Gaetano Valenti, Massimo Mancini. A decision support system (DSS) for traffic incident management in roadway tunnel infrastructure//Association for European Transport and contributors. 2006.

\section{DATA ABOUT THE AUTHORS}

Nikolaev Andrey Borisovich, Honoris Causa, Doctor of Technical Sciences, Professor, Head of Department

State Technical University - MADI

64, Leningradsky prospekt, Moscow, 125319, Russian Federation nikolaev.madi@mail.ru

Sapego Yuliya Sergeevna, Postgraduate Student, Department of «Automated Control Systems»

State Technical University - MADI 64, Leningradsky prospekt, Moscow, 125319, Russian Federation kafedra@asu.madi.ru 